\title{
Serum biomarkers for predicting pregnancy outcome in women undergoing IVF: human chorionic gonadotropin, progesterone, and inhibin A level at 11 days post-ET
}

\author{
Jee Hyun Kim', Mi Sun Shin', Gwang Yi', Byung Chul Jee ${ }^{1,2}$, Jung Ryeol Lee ${ }^{1,2}$, Chang Suk Suh ${ }^{1,2}$, Seok Hyun Kimm \\ Department of Obstetrics and Gynecology, 'Seoul National University Bundang Hospital, Seongnam; '2Seoul National University College of Medicine, \\ Seoul; ${ }^{3}$ Seoul National University Hospital, Seoul National University College of Medicine, Seoul, Korea
}

Objective: This study was performed to assess the prognostic value of serum $\mathrm{hCG}$, progesterone, and inhibin A levels measured at 11 days postET for predicting pregnancy outcome in women participating in IVF.

Methods: Between May 2005 and April 2008, sera were obtained from 70 infertile women who underwent IVF-ET at 11 days post-ET and stored. HCG, progesterone, and inhibin A levels were measured by commercial enzyme-linked immunosorbent assay kits. The predictive accuracy of hCG, progesterone, and inhibin A levels for establishment of intrauterine pregnancy and ongoing pregnancy was calculated by receiver-operating characteristic curve analysis.

Results: For the prediction of intrauterine and ongoing pregnancy, serum $\mathrm{hCG}$ was better than progesterone and inhibin A. The predictive performance of progesterone and inhibin A was similar. The serum progesterone and inhibin A levels were significantly correlated each other $(r=$ $0.915, p=0.010)$.

Conclusion: A single measurement of the serum hCG level is sufficient to predict pregnancy outcome in IVF-ET patients.

Keywords: Chorionic gonadotropin; Progesterone; Inhibins; In vitro fertilization; Embryo transfer; Outcomes, pregnancy

\section{Introduction}

Pregnancies achieved by IVF-ET are at increased risk of adverse outcomes compared with natural pregnancies or those achieved by ovum donation cycles [1]. Early pregnancy losses such as preclinical (chemical) and first trimester spontaneous abortions and ectopic pregnancies are common during the first trimester. Thus, the prediction of early pregnancy outcome, especially at the time of pregnancy test-

Received: Feb 26, 2012 - Revised: Mar 30, 2012 - Accepted: Mar 30, 2012 Corresponding author: Byung Chul Jee

Department of Obstetrics and Gynecology, Seoul National University Bundang Hospital, 82 Gumi-ro 173 beon-gil, Bundang-gu, Seongnam 463-707, Korea Tel: +82-31-787-7254 Fax:+82-31-787-4054 E-mail: blasto@snubh.org

This is an Open Access article distributed under the terms of the Creative Commons Attribution Non-Commercial License (http://creativecommons.org/licenses/by-nc/3.0/) which permits unrestricted non-commercial use, distribution, and reproduction in any medium, provided the original work is properly cited. ing, is of interest to many clinicians and infertile couples undergoing IVF-ET. Moreover, earlier diagnoses of complicated and poor pregnancy outcomes in patients that have undergone IVF would aid in counseling and management.

Ultrasound examination is part of the routine follow-up after IVF$\mathrm{ET}$, but the gestational sac is reliably visible after the third week after ET. To confirm the establishment of pregnancy and forecast its outcome at the earlier stage, research attempts have been made to identify serum biomarkers.

In practice, the initial establishment of pregnancy after ET is assessed by measuring serum $\mathrm{hCG}$ concentration which is reliable marker to forecast pregnancy outcome during the second week after ET. HCG is of placental origin, represents trophoblast functional integrity, and appears in maternal blood approximately 6 to 8 days after fertilization. Numerous studies have revealed that pregnancies with low ini- 
tial $h C G$ values are associated with a poor prognosis [2-6]. Serum hCG levels at 11 days post-ET are significantly lower for those that experience early pregnancy loss, and a cut-off of $50 \mathrm{mlU} / \mathrm{mL}$ was found to predict pregnancy outcome with good sensitivity and specificity of $75 \%$ and $81 \%$, respectively [4]. However, the positive predictive value (PPV) of hCG at this cut-off level was around $50 \%$ and its negative predictive value (NPV) was $93 \%$ in those study. However, in two recent studies, with cut-off values of 80 and $76 \mathrm{mlU} / \mathrm{mL}$, the PPV of hCG was $93.3 \%$ and $87 \%$ and NPV was $40 \%$ and $74 \%$, respectively $[2,3]$.

Since the corpus luteum is known to play an important role in early pregnancy, its activity could possibly be a marker of pregnancy outcome [7-9]. Progesterone is produced and secreted by the corpus luteum. Previous studies have revealed that progesterone is the most powerful single predictor of pregnancy outcome in natural conception $[10,11]$. However, the predictive role of progesterone in IVF pregnancy outcomes has been little studied and remains unproven $[6,9]$. Inhibins are gonadally-derived heterodimeric glycoproteins that are composed of an $a$ and $\beta_{A}$ or $\beta_{B}$, giving rise to inhibin $A$ and inhibin B. Inhibins are produced and secreted by the corpus luteum [12] and/ or placenta [13] in early pregnancy. Maternal serum levels of inhibin A are higher during the first trimester of pregnancy than in nonpregnant women [14]. The clearance of inhibin $A$ is fast, with a short halflife of 45 minutes [15].

In the present study, we investigated the role of progesterone and inhibin A measurement to discriminate among IVF pregnancy outcomes and compared the predictive value of progesterone and inhibin A with that achieved with hCG.

\section{Methods}

\section{Patients}

Between May 2005 and April 2008, sera were obtained and stored at the time of the pregnancy test (11 days post-ET) from 68 infertile women who underwent IVF-ET. The indications for IVF were tubal factor infertility $(n=28)$, unexplained infertility $(n=23)$, endometriosis $(n=8)$, male factor infertility $(n=6)$, and advanced maternal age $(\mathrm{n}=3)$. All patients received progesterone $(50 \mathrm{mg} /$ day intramuscularly, Watson Laboratories Inc., Morristown, NJ, USA) from the day of oocyte pick-up (OPU). Serum hCG level was measured at 11 days postET and the pregnancy outcomes were followed. This study was approved by the Institutional Review Board of Seoul National University Bundang Hospital (IRB No. B-1202-145-304).

\section{Hormone assays}

Serum hCG level was measured with a chemiluminescent microparticle immunoassay (Architect i2000, Abbott Laboratories, Abbott
Park, IL, USA) for total beta-hCG. The measuring range for $\mathrm{hCG}$ was 0.0 to $15,000 \mathrm{mIU} / \mathrm{mL}$. Samples with hCG concentration $>15,000$ $\mathrm{mIU} / \mathrm{mL}$ were diluted using normal saline and multiplication was performed by the dilution factor. The intra- and inter-assay coefficient of variation (CV) were $1.2 \%$ to $4.7 \%$ and $1.6 \%$ to $4.9 \%$, respectively.

Serum progesterone level measurement was performed using an electrochemiluminescence immunoassay (Elecsys Progesterone II; Hitachi High-technologies, Tokyo, Japan). The measuring range for progesterone was 0.03 to $60 \mathrm{ng} / \mathrm{mL}$. Samples with progesterone concentration $>60 \mathrm{ng} / \mathrm{mL}$ were diluted (1:10) using Elecsys Diluent Estradiol/Progesterone, and multiplication was performed by the dilution factor. The intra- and inter-assay CV were 1.5\% to 2.7\% and 4.1\% to $5.5 \%$, respectively.

Serum inhibin A was measured by a chemiluminescent immunoassay (Access Inhibin A; Beckman Coulter, Brea, CA, USA). The measuring range for inhibin $A$ was 1 to $1,500 \mathrm{pg} / \mathrm{mL}$. Samples with inhibin $A$ concentration $>1,500 \mathrm{pg} / \mathrm{mL}$ were diluted using Access Sample Diluent $A$ and multiplication was performed by the dilution factor. The intra- and inter-assay CV were $4.0 \%$ and $3.97 \%$, respectively.

\section{Pregnancy outcome}

Establishment of pregnancy was defined by an hCG level above 10 IU/L. Further classification of pregnancy outcomes was as follows: chemical pregnancy was defined as pregnancy detected by $\mathrm{hCG}$ measurement, for which no gestational sac had been seen on ultrasound; ongoing pregnancy was defined as progression beyond 12 weeks' gestation.

\section{Statistical analysis}

Data from 68 women were analyzed with SPSS ver.18 (SPSS Inc., Chicago, LL, USA) and MedCalc ver.12 (Medcalc Software, Mariakerke, Belgium). The Mann-Whitney $\mathrm{U}$ test was used for comparing continuous variables between groups. To assess the predictive accuracy of hormonal levels and compare the predictive accuracy, receiver-operating characteristic (ROC) curves and comparison of ROC curves were used. Pearson's correlation was used to examine the correlation between serum progesterone and inhibin A level. $p$-values $<0.05$ were considered statistically significant.

\section{Results}

Pregnancy outcomes were followed. The initial serum $\mathrm{hCG}$ titer was elevated in 29 women, but 11 women ended in chemical pregnancy. Intrauterine pregnancy was identified in 18 women. Among them, seven women experienced spontaneous miscarriage.

The age of the women in the ongoing pregnancy group was signif- 
Table 1. Age and the level of serum markers according to pregnancy outcome

\begin{tabular}{lcccc}
\hline & Non-pregnancy $(n=39)$ & Chemical pregnancy $(n=11)$ & Clinical abortion $(n=7)$ & Ongoing pregnancy $(n=11)$ \\
\hline Age of women & $34.3 \pm 3.5$ & $35.6 \pm 3.1$ & $36.7 \pm 2.6$ & $32.5 \pm 3.1$ \\
HCG (IU/L) & $3.2 \pm 0.6$ & $33.0 \pm 27.8$ & $261.6 \pm 226.2$ & $545.9 \pm 395.0$ \\
Progesterone $(\mathrm{ng} / \mathrm{mL})$ & $25.9 \pm 14.2$ & $39.5 \pm 23.3$ & $105.6 \pm 97.2$ & $74.3 \pm 56.4$ \\
Inhibin A $(\mathrm{pg} / \mathrm{mL})$ & $5.1 \pm 3.6$ & $37.5 \pm 63.6$ & $146.9 \pm 153.1$ & $122.7 \pm 115.3$ \\
\hline
\end{tabular}

Table 2. Efficiency of serum markers in predicting pregnancy outcomes

\begin{tabular}{|c|c|c|c|c|c|c|}
\hline & & & Cut-off & AUC (95\% Cl) & Sens. (\%) & Spec. (\%) \\
\hline \multirow[t]{6}{*}{ Overall $(n=68)$} & \multirow[t]{3}{*}{ Intrauterine pregnancy } & HCG (IU/L) & $>107$ & $0.998(0.000-1.000)$ & 88.9 & 100.0 \\
\hline & & Progesterone $(\mathrm{ng} / \mathrm{mL})$ & $>32.1$ & $0.767(0.609-0.926)$ & 77.8 & 62.0 \\
\hline & & Inhibin A (pg/mL) & $>6.75$ & $0.914(0.843-0.986)$ & 94.4 & 70.0 \\
\hline & \multirow[t]{3}{*}{ Ongoing pregnancy } & HCG (IU/L) & $>129.0$ & $0.971(0.000-1.000)$ & 100.0 & 93.0 \\
\hline & & Progesterone $(\mathrm{ng} / \mathrm{mL})$ & $>31.8$ & $0.732(0.543-0.921)$ & 81.8 & 56.1 \\
\hline & & Inhibin A (pg/mL) & $>6.9$ & $0.887(0.806-0.967)$ & 100.0 & 64.9 \\
\hline \multirow[t]{3}{*}{ HCG > $10 \mathrm{IU} / \mathrm{L}(\mathrm{n}=29)$} & \multirow[t]{3}{*}{ Ongoing pregnancy } & HCG (IU/L) & $>159.5$ & $0.909(0.000-1.000)$ & 100.0 & 83.3 \\
\hline & & Progesterone $(\mathrm{ng} / \mathrm{mL})$ & $>39.9$ & $0.606(0.388-0.825)$ & 72.7 & 55.6 \\
\hline & & Inhibin A (pg/mL) & $>44.4$ & $0.707(0.520-0.894)$ & 63.6 & 55.6 \\
\hline
\end{tabular}

AUC, area under curve; Sens., sensitivity; Spec., specificity.

icantly lower when compared with the clinical abortion group ( $p=$ 0.007), but the levels of the three serum markers in the two groups were similar (Table 1).

For the prediction of intrauterine pregnancy (clinical abortion+ongoing pregnancy), serum hCG (area under curve [AUC], 0.998; 95\% confidence interval $[\mathrm{Cl}], 0.000-1.000$ ), progesterone (AUC, 0.767; 95\% $\mathrm{Cl}, 0.609-0.926$ ) or inhibin A (AUC, $0.914 ; 95 \% \mathrm{Cl}, 0.834-0.986$ ) was a significant predictor (Table 2). However, the predictive accuracy of serum hCG was better than that of progesterone $(p=0.004)$ or inhibin $A(p=0.024)$. The predictive accuracy of progesterone and inhibin A were similar $(p=0.098)$.

For the prediction of ongoing pregnancy, serum hCG (AUC, 0.971; 95\% Cl, 0.000-1.000), progesterone (AUC, 0.732; 95\% Cl, 0.543-0.921), or inhibin A (AUC, $0.887 ; 95 \% \mathrm{Cl}, 0.806-0.967$ ) was also a significant predictor. The predictive accuracy of serum hCG was better than progesterone $(p=0.015)$ but similar to inhibin $\mathrm{A}(p=0.063)$. The predictive accuracy of progesterone and inhibin A were similar $(p=0.138)$. The serum progesterone and inhibin A levels were significantly correlated each other $(r=0.915, p=0.010)$.

Sub-group analysis was performed among 29 women in whom the serum hCG was above $10 \mathrm{IU} / \mathrm{L}$. The pregnancy in these women fell into one of three outcomes: chemical $(n=11)$, abortion $(n=7)$, or ongoing pregnancy $(n=11)$. To predict ongoing pregnancy, serum $\mathrm{hCG}$ (AUC, 0.909; 95\% Cl, 0.000-1.000) and inhibin A (AUC, 0.887; 95\% Cl, 0.806-0.967) were significant predictors, but serum progesterone (AUC, 0.606; 95\% Cl, 0.388-0.825) was not. Serum hCG was superior to progesterone $(p=0.015)$ but similar to inhibin $\mathrm{A}(p=0.070)$. The predictive accuracy of progesterone and inhibin A were similar ( $p=$ 0.491).

\section{Discussion}

We investigated three serum markers to predict pregnancy outcomes at the earliest stage. Like other studies, we found that the serum hCG level measured at 11 days post-ET is the best and most reliable biomarker for predicting IVF pregnancy outcome. Serum progesterone and inhibin A measurement has predictive value but plays a more limited role compared to serum hCG in revealing IVF pregnancy outcomes. Therefore, routine assessment of serum progesterone and inhibin A levels to predict pregnancy outcomes seems to be unwarranted.

loannidis et al. [9] studied the value of progesterone for prediction of pregnancy outcome in IVF cycles. At day 14 after OPU, maternal serum progesterone levels were found to predict pregnancy outcome after IVF cycles. Women with ongoing pregnancies had significantly higher serum progesterone levels (median, $430 \mathrm{nmol} / \mathrm{L}$ ) than those who had either an abnormal pregnancy, e.g., biochemical, miscarriage, or ectopic pregnancy $(72 \mathrm{nmol} / \mathrm{L})$ or failed to conceive $(33 \mathrm{nmol} / \mathrm{L})$. ROC analysis demonstrated that single serum progesterone on day 14 post-OPU (at a cut-off of $103 \mathrm{nmol} / \mathrm{L}$ ) could highly differentiate between normal and abnormal pregnancies (AUC, $0.927 ; 95 \% \mathrm{Cl}$, $0.89-0.96$; sensitivity $88.2 \%$, specificity $84 \%$ ). This finding indicates that serum progesterone levels are significantly elevated and can be used to detect women destined to have viable intrauterine pregnan- 
cies despite exogenous progesterone supplementation.

However, the present study revealed that progesterone has little importance in predicting IVF pregnancy outcomes among serum biomarkers. Our findings were consistent with the results from a previous study [6]. Several reasons for the low predictive accuracy of progesterone in forecasting IVF pregnancy outcomes are possible. First, a small number of women was included in this study; thus it could not reach adequate power. Second, GnRH agonist or antagonist treatment may influence the production of progesterone from the corpus luteum. Third, exogenous progesterone for luteal support may affect the potential efficacy of serum progesterone in predicting outcomes. In our data, the progesterone level was higher than that of natural pregnancy in all pregnancy outcomes including non-pregnancy. Elevated serum progesterone due to exogenous progesterone in the IVF cycle may decrease the predictive power of discriminating between ongoing pregnancies and clinical abortions.

Initially low levels and a rapid decline of serum inhibin A concentrations during the first trimester have been shown to be related to nonviable pregnancies [16]. However, in a subsequent study, no significant difference was found between serum inhibin A levels at day 16 post-OPU in those with ongoing pregnancies and those that spontaneously aborted [8]. Nevertheless, inhibin A levels were shown to predict preclinical abortions after IVF [17]. Two recent reports indicated that inhibin $A$ is superior to hCG for the prediction of preclinical abortion, but no significant differences were observed between the predictive values of hCG, inhibin A, or their combination in terms of differentiating between ongoing pregnancies and early pregnancy losses $[8,17]$.

In this study, inhibin A level in the ongoing pregnancy group was not significantly different than that of the clinical abortion group $(122.7 \pm 115.3$ vs. $146.9 \pm 153.1)$. The cut-off level of inhibin A (AUC, $0.914 ; 95 \% \mathrm{Cl}, 0.834-0.986)$ was $6.75 \mathrm{pg} / \mathrm{mL}$ for the prediction of intrauterine pregnancy, whereas for the prediction of ongoing pregnancy among women in whose pregnancy was established, the cutoff level increased to $44.4 \mathrm{pg} / \mathrm{mL}$ and predictive accuracy decreased to 0.707 . These findings suggest that a low level of inhibin $A$ at the time of very early pregnancy indicates the likelihood of a poor outcome, but forthcoming abortions are not predictable by early inhibin A measurement.

Serum concentrations of progesterone, estradiol, and hCG measured 12 to 13 days post-ET were found to be higher in an ongoing pregnancy group than in an abortion group [18]. However, the best predictor of ongoing pregnancy was found to be hCG concentration. Moreover, this multiple marker strategy was unhelpful in distinguishing viable from non-viable pregnancies.

Multiple hCG level measurements continue to be performed on IVF patients to monitor pregnancy progress. The prognostic values of single and paired measurements of serum hCG levels were analyzed in IVF setting $[11,12]$. An earlier report demonstrated that a single hCG determination on day 15 post-ET combined with the ratio of hCG on day 22 vs. day 15 has a higher diagnostic accuracy for predicting pregnancy outcome than absolute levels on either of these two days [19]. However, a recent report found that serial hCG measurements do not contribute to the identification of non-ongoing pregnancies. A single hCG determination enabled approximately $40 \%$ of non-viable pregnancies to be identified with a $85 \%$ positive predictive value, and the serial testing was found to have a $70 \%$ positive predictive value for identifying non-ongoing pregnancies [20].

We concluded that the serum hCG level is clinically valuable for forecasting pregnancy outcome after IVF-ET. Further research is needed to identify biomarkers discriminating ectopic pregnancies.

\section{Conflict of interest}

No potential conflict of interest relevant to this article was reported.

\section{References}

1. Simón C, Landeras J, Zuzuarregui JL, Martín JC, Remohí J, Pellicer A. Early pregnancy losses in in vitro fertilization and oocyte donation. Fertil Steril 1999;72:1061-5.

2. Ochsenkühn R, Arzberger A, von Schönfeldt V, Engel J, Thaler CJ, Noss $\mathrm{U}$. Predictive value of early serum beta-hCG levels after single blastocyst transfer. Acta Obstet Gynecol Scand 2009;88:1382-8.

3. Poikkeus P, Hiilesmaa V, Tiitinen A. Serum HCG 12 days after embryo transfer in predicting pregnancy outcome. Hum Reprod 2002;17:1901-5.

4. Urbancsek J, Hauzman E, Fedorcsák P, Halmos A, Dévényi N, Papp Z. Serum human chorionic gonadotropin measurements may predict pregnancy outcome and multiple gestation after in vitro fertilization. Fertil Steril 2002;78:540-2.

5. Papageorgiou TC, Leondires MP, Miller BT, Chang AS, Armstrong $A B$, Scott $L A$, et al. Human chorionic gonadotropin levels after blastocyst transfer are highly predictive of pregnancy outcome. Fertil Steril 2001;76:981-7.

6. Homan G, Brown S, Moran J, Homan S, Kerin J. Human chorionic gonadotropin as a predictor of outcome in assisted reproductive technology pregnancies. Fertil Steril 2000;73:270-4.

7. Treetampinich C, $O^{\prime}$ Connor AE, MacLachlan V, Groome NP, de Kretser DM. Maternal serum inhibin A concentrations in early pregnancy after IVF and embryo transfer reflect the corpus luteum contribution and pregnancy outcome. Hum Reprod 2000; 15:2028-32.

8. Hauzman E, Fedorcsák P, Klinga K, Papp Z, Rabe T, Strowitzki T, et 
al. Use of serum inhibin A and human chorionic gonadotropin measurements to predict the outcome of in vitro fertilization pregnancies. Fertil Steril 2004;81:66-72.

9. Ioannidis G, Sacks G, Reddy N, Seyani L, Margara R, Lavery S, et al. Day 14 maternal serum progesterone levels predict pregnancy outcome in IVF/ICSI treatment cycles: a prospective study. Hum Reprod 2005;20:741-6.

10. Elson J, Salim R, Tailor A, Banerjee S, Zosmer N, Jurkovic D. Prediction of early pregnancy viability in the absence of an ultrasonically detectable embryo. Ultrasound Obstet Gynecol 2003;21: 57-61.

11. Chetty M, Sawyer E, Dew T, Chapman AJ, Elson J. The use of novel biochemical markers in predicting spontaneously resolving 'pregnancies of unknown location.' Hum Reprod 2011;26:131823.

12. Yamoto M, Minami S, Nakano R. Immunohistochemical localization of inhibin subunits in human corpora lutea during menstrual cycle and pregnancy. J Clin Endocrinol Metab 1991;73:470-7.

13. Petraglia F, Garuti GC, Calza L, Roberts V, Giardino L, Genazzani $A R$, et al. Inhibin subunits in human placenta: localization and messenger ribonucleic acid levels during pregnancy. Am J Obstet Gynecol 1991;165:750-8.

14. Muttukrishna S, George L, Fowler PA, Groome NP, Knight PG. Measurement of serum concentrations of inhibin-A (alpha-beta A di- mer) during human pregnancy. Clin Endocrinol (Oxf) 1995;42: 391-7.

15. Muttukrishna S, Child TJ, Groome NP, Ledger WL. Source of circulating levels of inhibin A, pro alpha C-containing inhibins and activin A in early pregnancy. Hum Reprod 1997;12:1089-93.

16. Lockwood GM, Ledger WL, Barlow DH, Groome NP, Muttukrishna S. Measurement of inhibin and activin in early human pregnancy: demonstration of fetoplacental origin and role in prediction of early-pregnancy outcome. Biol Reprod 1997;57:1490-4.

17. Hauzman E, Murber A, Fancsovits P, Papp Z, Urbancsek J. Use of biochemical markers to predict the outcome of pregnancies conceived by in vitro fertilization. Orv Hetil 2006;147:1409-20.

18. Carmona F, Balasch J, Creus M, Fabregues F, Casamitjana R, Civico $\mathrm{S}$, et al. Early hormonal markers of pregnancy outcome after in vitro fertilization and embryo transfer. J Assist Reprod Genet 2003;20:521-6.

19. Chen CD, Ho HN, Wu MY, Chao KH, Chen SU, Yang YS. Paired human chorionic gonadotrophin determinations for the prediction of pregnancy outcome in assisted reproduction. Hum Reprod 1997;12:2538-41.

20. Alahakoon TI, Crittenden J, Illingworth P.Value of single and paired serum human chorionic gonadotropin measurements in predicting outcome of in vitro fertilisation pregnancy. Aust N Z J Obstet Gynaecol 2004;44:57-61. 